\title{
Practical assessment of the quantification of atherosclerotic lesions in $\operatorname{apoE}^{-/-}$mice
}

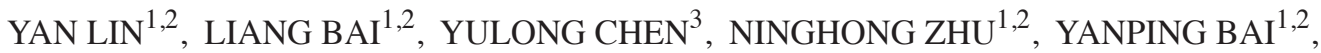 \\ QIANWEI LI ${ }^{1,2}$, SIHAI ZHAO ${ }^{1,2}$, JIANGLIN FAN $^{4}$ and ENQI LIU ${ }^{1-3}$ \\ ${ }^{1}$ Laboratory Animal Center, School of Medicine, Xi'an Jiaotong University; ${ }^{2}$ Research Institute of Atherosclerotic Disease, \\ Xi'an Jiaotong University, Xi'an, Shaanxi 710061; ${ }^{3}$ Shaanxi Key Laboratory of Ischemic Cardiovascular Disease, \\ Institute of Basic and Translational Medicine, Xi'an Medical University, Xi'an, Shaanxi 710021, P.R. China; \\ ${ }^{4}$ Department of Molecular Pathology, Interdisciplinary Graduate School of Medicine and Engineering, \\ University of Yamanashi, Yamanashi 409-3898, Japan
}

Received September 26, 2014; Accepted June 18, 2015

DOI: $10.3892 / \mathrm{mmr} .2015 .4084$

\begin{abstract}
Genetic manipulations have enabled the mouse to be widely used as an animal model for investigating the mechanisms of human atherosclerotic disease. However, there is no standard method for quantifying atherosclerotic lesions among different laboratories. The present study introduces a thorough and precise quantitative assessment of atherosclerotic lesions in mice. In the present study, 6-week-old apoE $\mathrm{E}^{-/-}$mice were fed either a chow diet or a high-fat diet (HFD) for 12 weeks. Plasma lipid levels were measured every four weeks. Aortic atherosclerotic lesions were quantified and analyzed using an image analysis system. The aortic tree was isolated and stained with Oil Red $\mathrm{O}$ to measure the gross lesion area. The heart was removed and divided into sequential cross sections, which were then assessed for microscopic intimal lesions in the aortic root as follows: (1) Elastic van Gieson staining was performed to determine the area of the atherosclerotic lesion; (2) cross sections were stained with hematoxylin and eosin for histological analysis; and (3) cross sections were stained with Oil Red $\mathrm{O}$ and immunohistochemical staining for quantitative analysis of the cellular components within the lesions. $\mathrm{ApoE}^{-/-}$mice fed with either the chow diet or HFD developed severe atherosclerosis in the aortic root, however, there were few lesions in the remainder of the aortic tree. Compared
\end{abstract}

Correspondence to: Professor Enqi Liu, Research Institute of Atherosclerotic Disease, Xi'an Jiaotong University, 76 Yanta West Road, Xi'an, Shaanxi 710061, P.R. China

E-mail: liuenqi@mail.xjtu.edu.cn

Abbreviations: HFD, high-fat diet; AUC, incremental area under the curve; EVG, elastic van Gieson; HFD, high-fat diet; H\&E, hematoxylin and eosin; OCT, ornithine carbamoyltransferase; PBS, phosphate-buffered saline; TC, total cholesterol; TG, triglyceride; VSMCs, vascular smooth muscle cells

Key words: aorta, atherosclerosis, morphometry, mouse with the control group, the HFD apoE $\mathrm{E}^{-/-}$mice had increased plasma lipid levels and increases in the gross lesion area in the aortic tree, the microscopic lesion area in the aortic root and the number of macrophages, vascular smooth muscle cells and neutral lipids present within the lesions. HFD feeding in the apoE ${ }^{-/-}$mice accelerated the development of atherosclerosis. The quantitative method described in the present study may be used to assist in future investigations of atherosclerosis in mice.

\section{Introduction}

The most commonly used species in contemporary animal models is the mouse, which has been used in the investigation of the development and progression of atherosclerotic lesions (1). The mouse offers several advantages as an animal model, including having a well-known genetic background, being easy to breed and having a low maintenance cost. However, its small size and certain physiological characteristics are often considered limiting factors (2). For example, the plasma lipoprotein profile in mice is very different from that in humans. The circulating cholesterol is predominantly packaged in high density lipoprotein particles in mice, whereas it is packaged in low density lipoprotein particles in humans. One reason for this lipoprotein profile difference is the absence of the cholesterol-ester transfer protein in mice. Another difference between mice and humans is their response to dietary cholesterol; mice do not absorb dietary cholesterol, whereas humans absorb $\sim 50 \%$ of dietary cholesterol (2). In addition, wild-type mice are notoriously resistant to the development of atherosclerotic lesions (3). At present, mouse models for atherosclerosis are dependent on genetic modifications of lipoprotein metabolism and additional dietary alterations (4). $\mathrm{ApoE}^{-/-}$mice were the first genetically modified mice that developed atherosclerosis spontaneously and, have thus been widely used in the investigation of atherosclerotic disease $(5,6)$.

Quantifying atherosclerotic lesions is a stable and reliable indicator of the severity of atherosclerosis (7). Atherosclerotic plaques can form in the carotid artery, pulmonary artery, femoral artery, aortic root and brachiocephalic artery (8). All 
of these anatomical sites can be used to quantify atherosclerotic lesions $(7,8)$. Due to the fact that the aortic root in mice is the site with the greatest proclivity to develop lesions, several laboratories use plaque formation in the aortic root to evaluate the severity of atherosclerosis in mice (9-12). This method was first described by Paigen et al (11), who used sequential cross sections through the heart and aortic root to quantify the lesion area in a constant number of cross sections, beginning with the aortic valve leaflet. Due to the fact that variability may occur in the aortic root, certain studies have extended the sectioned distance by more than $1 \mathrm{~mm}$, to increase the accuracy of the method $(12,13)$. This method is successful, but labor-intensive. Additionally, atherosclerotic plaques can form anywhere in the entire arterial tree, thus, the assessment of atherosclerosis in the aortic root alone is not sufficient to evaluate the severity of atherosclerosis $(8,14)$. For improved accuracy, the assessment requires complementation by the determination of the gross lesion area in the remainder of the arterial tree. The morphometric method used to quantify the gross lesion area in the arterial tree has been described in detail previously (14). The combination of these two indicators may offer an improved method to evaluate the severity of atherosclerosis. The aim of the present study was to introduce, in detail, a more practical method for the systematic analysis of aortic atherosclerosis in mice, and to use this method to analyze the effect of a high-fat diet (HFD) on the development of atherosclerosis in $\mathrm{apoE}^{-/}$ mice.

\section{Materials and methods}

Animals and diets. The apoE ${ }^{-/-}$mice with a C57BL/6J background were obtained from the Laboratory Animal Centre of Xi'an Jiaotong University (School of Medicine, Xi'an, China). All animals were maintained in cages of three of four animals in a temperature controlled $\left(20 \pm 2^{\circ} \mathrm{C}\right)$ facility with a 12-h day/night cycle and free access to food and water. A total of 206 -week old, apoE ${ }^{-/-}$male mice were randomly divided into a control group and a HFD group $(n=10$ for each group). The control mice were fed a standard chow diet for 12 weeks. The mice in the HFD group were fed a HFD, containing $21 \%$ fat and $0.15 \%$ cholesterol. The chow diet and HFD were provided by Beijing Ke'ao Xieli Feed Co., Ltd. (Beijing, China). The animals and their feed were weighed every week. All experimental procedures involving the use of animals were approved by the Laboratory Animal Administration Committee of Xi'an Jiaotong University and performed, according to the Xi'an Jiaotong University Guidelines for Animal Experimentation. The present study was performed in accordance with the 'Guide for the Care and Use of Laboratory Animals' (15).

Plasma lipid measurements. Blood samples were obtained on weeks $0,4,8$ and 12, and were collected into heparin-coated capillary glass tubes from the mouse tail vein following $8 \mathrm{~h}$ of fasting. The experimental period was 12 weeks. The blood samples were stored on ice and centrifuged ( $876 \mathrm{x} \mathrm{g}, 20 \mathrm{~min}$, $4^{\circ} \mathrm{C}$ ) to obtain plasma. The plasma levels of total cholesterol (TC) and triglyceride (TG) were measured using standard commercial assay kits (Biosino Bio-Technology and Science, Inc., Beijing, China) (16).
Separation of the heart and arterial tree. At the end of the experiment, all of the mice were placed and fixed in a supine position under anesthesia using pentobarbital sodium (40 mg/kg body weight, intraperitoneal; Sigma-Aldrich, St. Louis, MO, USA). A ventral midline incision was made from the mandible to the pelvic area, and the skin, subcutaneous tissue and diaphragm were separated to expose the chest and abdomen. Phosphate-buffered saline (PBS; $10 \mathrm{ml} ; 0.01 \mathrm{M}$ ) was injected into the systemic circulation over $5 \mathrm{~min}$ via an infusion needle, which was inserted into the left ventricle from the apex of the heart (Fig. 1A). The perfusion liquid was then discharged via an incision in the right atrial appendage. The liver and kidney gradually turned white in appearance from the perfusion. For quantification of the atherosclerotic lesions in the aortic tree, the entire aorta, between the heart and iliac arteries was dissected. Initially, the intestines, liver, kidney, stomach, septum transversum and other redundant structural tissues were removed, in order to expose the heart and aorta. To isolate the aortic arch and its branch arteries, the lungs and esophagus were also removed. Subsequently, the heart was lifted at the apex using forceps, and the left and right carotid arteries and left subclavian artery were severed close to the spine. The remainder of the aorta was separated along the spine until the iliac arteries were free. The entire aorta was immersed overnight in a $20 \%$ sucrose solution. The muscle, adipose tissue and the adventitia around the aorta were removed under a dissecting microscope (SZX7; Olympus Corporation, Tokyo, Japan) (Fig. 1B). The heart with attached 2-3 $\mathrm{mm}$ long aortic arch was dissected and fixed on a foam panel with a syringe needle. The lower $\sim 70 \%$ of the ventricular mass was cut away, as shown in Fig. 1C. The upper cardiac portion was embedded in ornithine carbamoyltransferase (OCT; Sakura Finetek Japan Co., Ltd., Tokyo, Japan), with the aortic arch positioned upwards using a metal mold (Fig. 1D), and immediately frozen in liquid nitrogen and stored at $-80^{\circ} \mathrm{C}$ for later use. The remaining arterial tree between the aortic arch and iliac arteries was fixed in $4 \%$ paraformaldehyde (Sigma-Aldrich) until use.

Oil Red $O$ staining of the arterial tree. Prior to staining of the arterial tree with Oil Red O (Sigma-Aldrich), the stock solution (1 g Oil Red O powder dissolved in $200 \mathrm{ml}$ isopropyl alcohol (Tianjin Tianli Chemical Reagents Ltd., Tianjin, China), thoroughly mixed) and working solution (3:2 stock solution:double distilled water, filtered at room temperature) were prepared. The working solution required use within two hours of preparation. The fixed arterial trees were washed three times in double distilled water ( $1 \mathrm{~min} /$ wash $)$ and then immersed in $60 \%$ isopropyl alcohol for $5 \mathrm{~min}$. The arterial trees were subsequently incubated in the Oil Red $\mathrm{O}$ solution at $37^{\circ} \mathrm{C}$ for $30 \mathrm{~min}$. Subsequent to staining, the samples were briefly immersed into $60 \%$ isopropyl alcohol solution and then washed with double distilled water. The atherosclerotic lesions were stained in red with Oil Red O.

Quantification of the gross lesion area. Images of the Oil Red O-stained arterial trees were captured for measurement of the area of the aortic en face lesions. The arterial tree stained with Oil Red $\mathrm{O}$ was opened longitudinally between the aortic arch and the iliac arteries. The first incision was along 
A

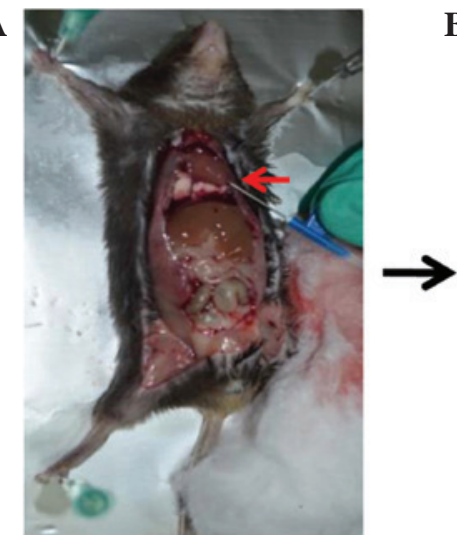

B

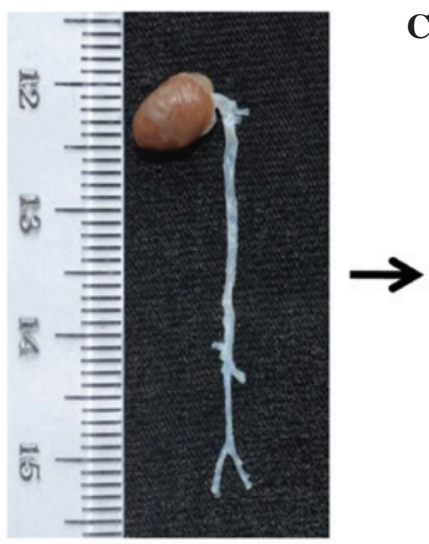

C

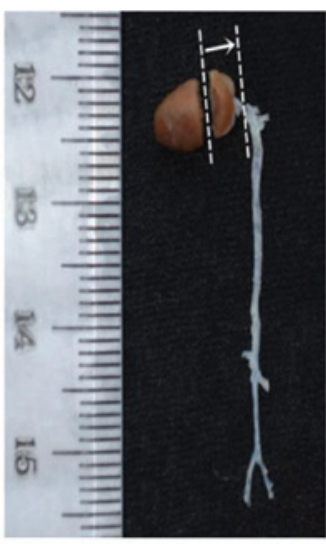

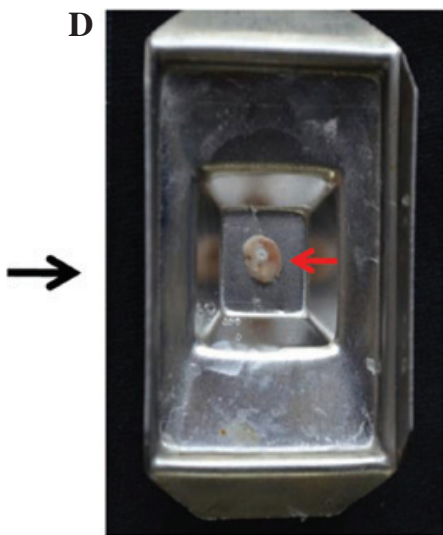

Figure 1. Method of preparing aortic tissues. (A) Mouse perfusion. Red arrow indicates the apex of the heart. (B) Separation of the heart and the entire arterial tree. (C) Preparation of the aortic root. The heart tissue between the two dotted lines was sliced, with the direction of slicing indicated by the white arrow, and the (D) upper half of the heart with the aortic root was embedded in ornithine carbamoyltransferase, with the aortic arch positioned upwards. Red arrow indicates aortic arch.

the ventral side of the aorta and inner curvature of the aortic arch. The second incision was along the outer curvature of the aortic arch, in order to flatten the arteries for imaging (14). Images of the arteries were captured using a digital camera (D90, Nikon Corporation, Tokyo, Japan) against a wet black cloth with an adjacent ruler. The digital images of the entire Oil Red $\mathrm{O}$ stained arterial trees were evaluated using image analysis software (WinROOF, version 6.5; Mitani Co., Ltd. Fukui, Japan) (17). The lesions on the arterial tree were expressed as a percentage of the area of lesions (red), relative to the surface area of the entire aorta (18). All measurements were performed independently by two observers in a blinded-manner.

Serial cross-sectioning of the aortic root. Subsequent to washing with PBS, the OCT-embedded upper cardiac portion, with an attached 2-3 mm long aortic arch, was fixed on the specimen dish with the arch positioned downwards. The specimen dish was subsequently installed into a specimen holder for serial sectioning. Sequential cross sections were sliced using a Leica CM1950 Cryostat (Leica Microsystems, Wetzlar, Germany) between the base of the heart and aortic arch, until the first aortic valve leaflet appeared (Fig. 2A). Subsequently, $8-\mu \mathrm{m}$ thick cross sections were sectioned until three aortic valve leaflets appeared together (Fig. 2B). The distance from where the first aortic valve leaflet appeared, to where all three of the aortic valve leaflets appeared together did not exceed $80 \mu \mathrm{m}$. The first $8-\mu \mathrm{m}$ thick section with the three aortic valve leaflets was mounted onto a glass slide. The adjoining eight sections, including the first section, were collected one by one onto eight glass slides, respectively, marked in order between numbers one and eight. The ninth section was collected onto the first glass slide, the tenth section was collected onto the second glass slide, and so forth, until each of the eight glass slides held eight sections. The three aortic valve leaflets were expected to be incomplete on the final cross section (Fig. 2C and D). The eight frozen sections collected on each of the glass slides covered a distance of $512 \mu \mathrm{m}$. All the sequential sections were stored at $-80^{\circ} \mathrm{C}$ until use, following which the eight glass slides were stained.
Quantification of microscopic lesions in the aortic root. For quantification of microscopic lesions in the aortic root, one of the glass slides of the frozen cross sections was stained with elastic van Gieson (EVG) $(16,19)$. The Verhoeff hematoxylin solution, $2 \% \mathrm{FeCl}_{3}$ solution and $5 \%$ sodium thiosulfate solution (all Maiwei Biotechnology Ltd., Xiamen, China) were prepared in advance. Verhoeff hematoxylin was produced using two a 2:4:4 ratio of $5 \%$ ethanol hematoxylin solution ( $2 \mathrm{~g}$ hematoxylin powder dissolved in $40 \mathrm{ml}$ ethanol at $50^{\circ} \mathrm{C}$, which was then filtered and stored overnight prior to use), $10 \% \mathrm{FeCl}_{3}$ solution ( $10 \mathrm{~g} \mathrm{FeCl}_{3}$ dissolved in $100 \mathrm{ml}$ double distilled water) and four parts Lugol's iodine solution ( $2 \mathrm{~g}$ iodine powder and $1 \mathrm{~g}$ potassium iodide powder dissolved successively in $100 \mathrm{ml}$ double distilled water), which were mixed in successively.

Following maintenance at room temperature for a minimum of $30 \mathrm{~min}$, the glass slides with eight frozen aortic root cross sections were fixed in acetone (100\%; Sigma-Aldrich) for $10 \mathrm{~min}$, incubated in Verhoeff hematoxylin solution for $15 \mathrm{~min}$ at room temperature and then immersed in $2 \% \mathrm{FeCl}_{3}$ solution ( $2 \mathrm{~g} \mathrm{FeCl}_{3}$ dissolved in $100 \mathrm{ml}$ double distilled water) for $10 \mathrm{sec}$. The fibrous tissues were stained black on a gray background. Subsequent to deiodination with a $5 \%$ sodium thiosulfate solution for $1 \mathrm{~min}$, the sections were counterstained with collagen VG staining fluid (Maiwei Biotechnology Ltd.) for $40 \mathrm{sec}$ and immersed in $95 \%$ ethanol for $1 \mathrm{~min}$. The sections were rinsed with tap water between each step. Following dehydration with ethanol and coverslipping with dimethylbenzene (Xi'an Chemical Reagent Factory, Xi'an, China), the elastic fibers exhibited a black appearance, indicating the junction between the tunica intima and tunica media. Images of all the EVG-stained slides were captured using a Nikon DS-Ril digital camera attached to a Nikon Eclipse 80i light microscope (Nikon Corporation) and analyzed using the image analysis software described above. The intimal lesion size was expressed as $\mathrm{mm}^{2}$.

Quantification of neutral lipids in the plaques of the aortic root. For quantification of the neutral lipids in the plaques of the aortic root, one of the remaining glass slides containing frozen cross sections was stained with Oil Red O. The detailed 

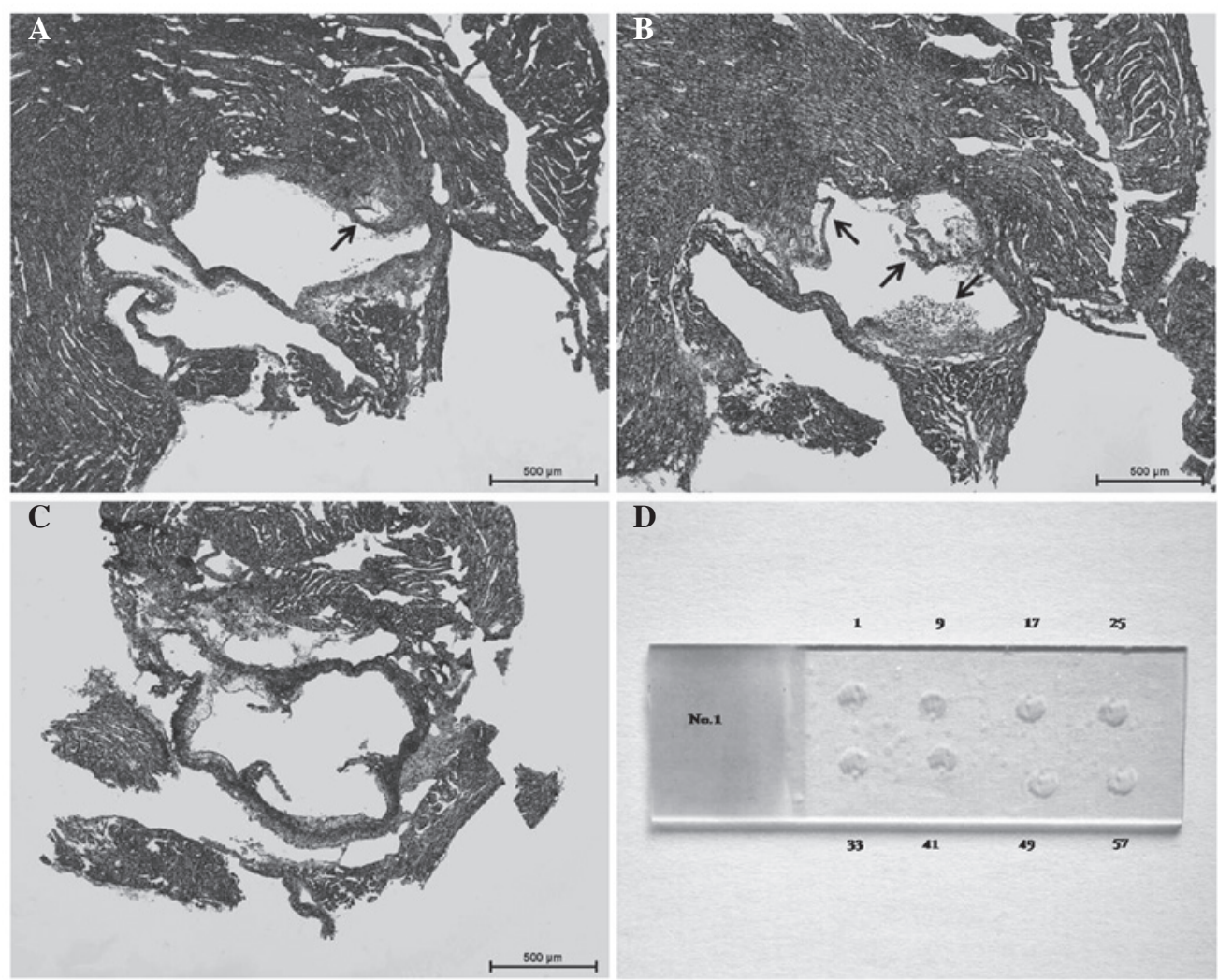

Figure 2. Serial cross-sectioning of the aortic root. (A) A cross section, in which one of the aortic valve leaflets first appeared. (B) First 8- $\mu \mathrm{m}$ thick cross section, in which the three aortic valve leaflets first appeared together. (C) Last 8- $\mu$ m thick cross section, in which the three aortic valve leaflets in the cross section were incomplete. (D) First glass slide containing eight frozen cross-sections with $64 \mu \mathrm{m}$ intervals. Numbers represent the sequence of serial frozen cross-sections.

steps of Oil Red O staining are described above. The neutral lipids in the plaques were stained red. Images of all the Oil Red O-stained slides were captured using a Nikon DS-Ril digital camera attached to the Nikon Eclipse 80i (Nikon Corporation) light microscope, and analyzed using the image analysis software, described above. Positive-labeling (red) was defined by the application of a color threshold mask, and the same threshold was applied to all sections (18). The neutral lipid area size was expressed as $\mathrm{mm}^{2}$.

Analysis of the composition of the atherosclerotic plaques of the aortic root. For microscopic evaluation of the cellular components, two of the other glass slides containing frozen sections were immunohistochemically stained with antibodies against smooth muscle $\alpha$-actin (rabbit polyclonal; cat. no. ab5694; Abcam, Cambridge, MA, USA) and macrophage galectin-3 (rabbit polyclonal; cat. no. ab53082; Abcam) as previously described (18). Galectin-3 (galactoside-binding protein, Mac-2 antigen) is a member of the Galectin family of carbohydrate-binding proteins. Galectin-3 is often pro-inflammatory and is involved in innate immunity; it is widely expressed in macrophages (20). The glass slides were placed at room temperature for a minimum of 30 min following removal from the cryogenic refrigerator. They were then fixed in $4 \%$ paraformaldehyde for $15 \mathrm{~min}$ and incubated with $3 \%$ hydrogen peroxide (Sigma-Aldrich) to inhibit endogenous peroxidase activity. The frozen sections were then incubated with $5 \%$ normal goat serum to inhibit non-specific binding. The frozen sections were incubated with the primary antibody at $4^{\circ} \mathrm{C}$ overnight and then incubated with the horseradish peroxidase-conjugated goat anti-rabbit immunoglobulin $\mathrm{G}$ secondary antibody (1:200; cat. no. D0487; Dako, Glostrup, Denmark) at $37^{\circ} \mathrm{C}$ for $60 \mathrm{~min}$. The labelling of the smooth muscle $\alpha$-actin and macrophages were visualized using 3,3'-diaminobenzidine (Sigma-Aldrich, Tokyo, Japan) and the glass slides were rinsed with PBS between each step. Following dehydration with ethanol and coverslipping with dimethylbenzene, images of all the immunolabeled sections were captured under a light microscope camera system, described above, and analyzed using the same software. Positive labelling (brown) was defined by the application of a color threshold mask, and the same threshold was applied to all sections (18). The results are expressed as $\mathrm{mm}^{2}$. The cross sections were also stained with hematoxylin and eosin (H\&E) for histological analysis.

Statistical analysis. All the results are expressed as the mean \pm standard deviation. The two groups were compared using Student's t-test for the data that had an equal F-value and Welch's t-test for the data with an unequal F-value. $\mathrm{P}<0.05$ was considered to indicate a statistically significant difference. Data analysis was performed using the SPSS software, version 13.0 (SPSS, Inc., Chicago, IL, USA).

\section{Results}

Biochemical parameters. Plasma lipid levels were measured in all the mice in the two groups every four weeks of the 

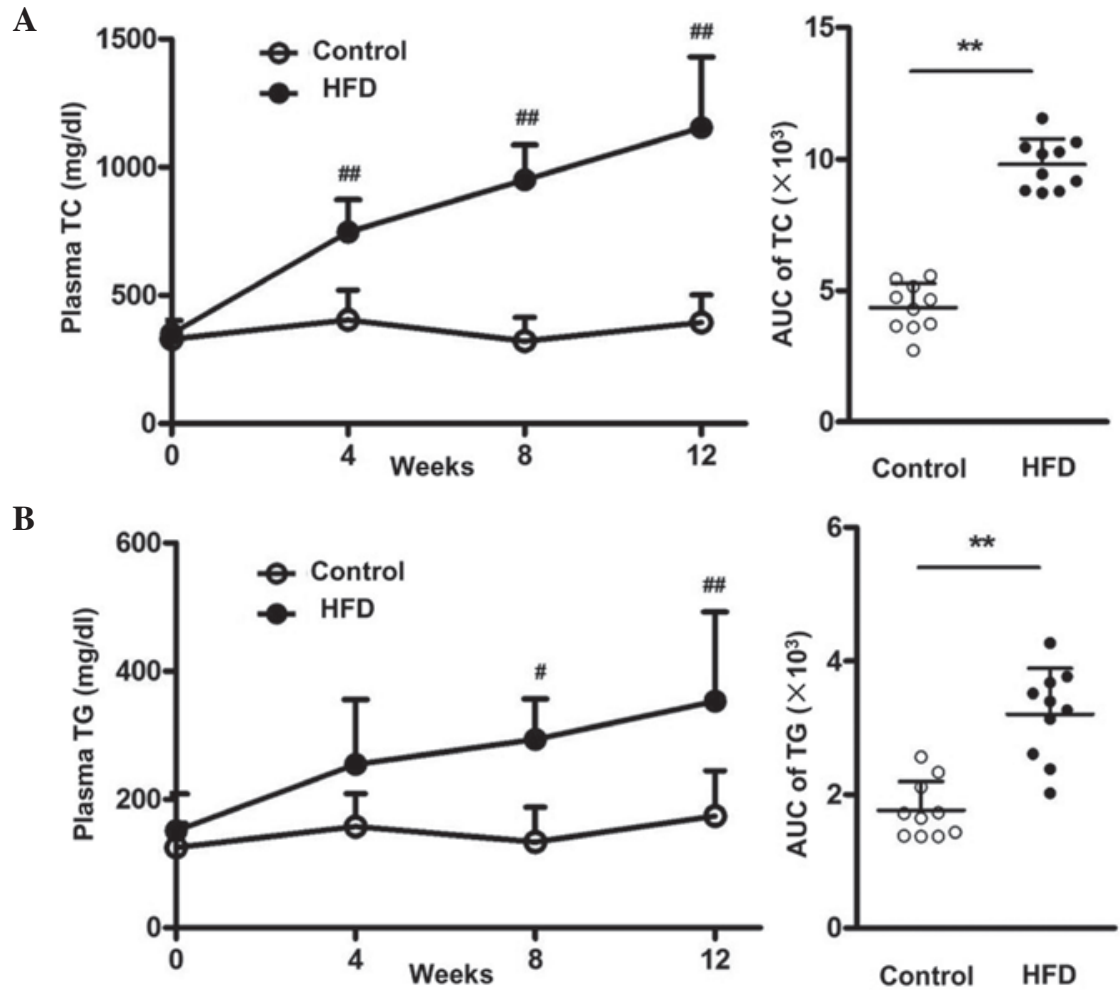

Figure 3. Plasma lipid levels. (A) Plasma TC in the HFD-fed and control mice. (B) Plasma TG in the HFD-fed and control mice. Data are expressed as the mean \pm standard deviation ( $\mathrm{n}=10$ for each group). ${ }^{* *} \mathrm{P}<0.01$, vs. control; ${ }^{\#} \mathrm{P}<0.05$ and ${ }^{\# \#} \mathrm{P}<0.01$, vs. week 0 . TC, total cholesterol; HFD, high-fat diet; TG, triglyceride; AUC, area under the curve.

A

\section{Control}

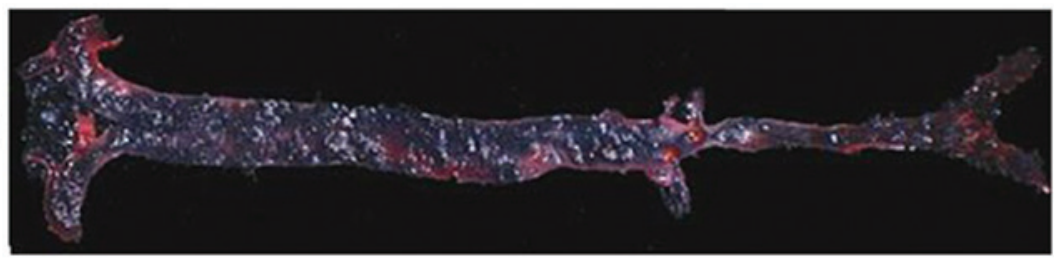

HFD

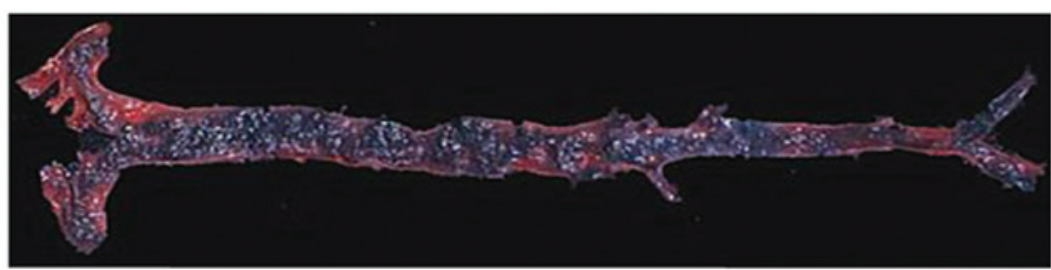

B

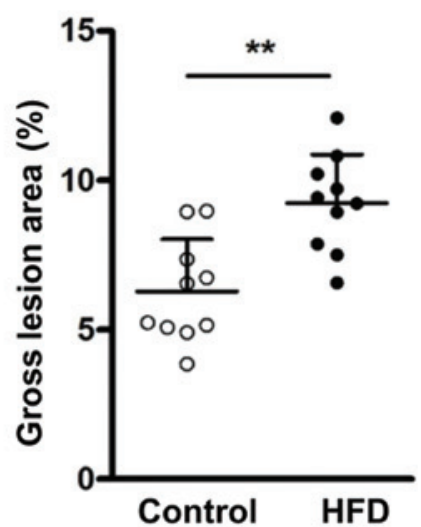

Figure 4. Gross lesions of the entire arterial tree. (A) Representative images of gross atherosclerotic lesions in HFD-fed and control mice, stained with Oil Red O. (B) Results of the sizes of the gross lesion areas in the entire arterial tree, compared with control mice. Data are expressed as the mean \pm standard deviation ( $\mathrm{n}=10$ for each group). ${ }^{* *} \mathrm{P}<0.01$, vs. control. HFD, high-fat diet.

experimental period. In order to compare the plasma lipid levels between the two groups during the 12 weeks, the incremental AUC was calculated using the trapezium rule (21). As shown in Fig. 3, the plasma levels of TC and TG levels in the apoE $E^{-/}$mice following 12 weeks exposure to HFD increased significantly, compared with the levels at week $0 \quad(\mathrm{P}<0.01$; Fig. 3), while no significant differences in the control group. In the HFD group of apoE-/- mice, the plasma TG levels were not significantly different at week $4(\mathrm{P}=0.093)$, as compared with the levels of plasma TG at week 0 ; however, at week 8 the plasma TG levels were significantly increased $(\mathrm{P}=0.011)$. The plasma concentrations of TC and TG in the apoE $\mathrm{E}^{-/-}$mice of the HFD group were also higher than in the control group for the duration of the experiment $(\mathrm{P}<0.01$; Fig. 3$)$.

Assessment of gross atherosclerotic lesion area. Following Oil Red $\mathrm{O}$ staining, atherosclerotic lesions were grossly observed in the aortic tree of apoE $\mathrm{E}^{-/-}$mice in the two groups. The lesions developed in the aortic root and were also sporadically present throughout the aorta and its principal branches (Fig. 4A). The ratio of the total area of atherosclerotic lesions, relative to that the luminal surface area of the entire aorta was higher in the HFD group of $a p o E^{-/-}$mice, compared with the control group of mice on a chow diet $(\mathrm{P}<0.01$, Fig. 4B). Feeding 


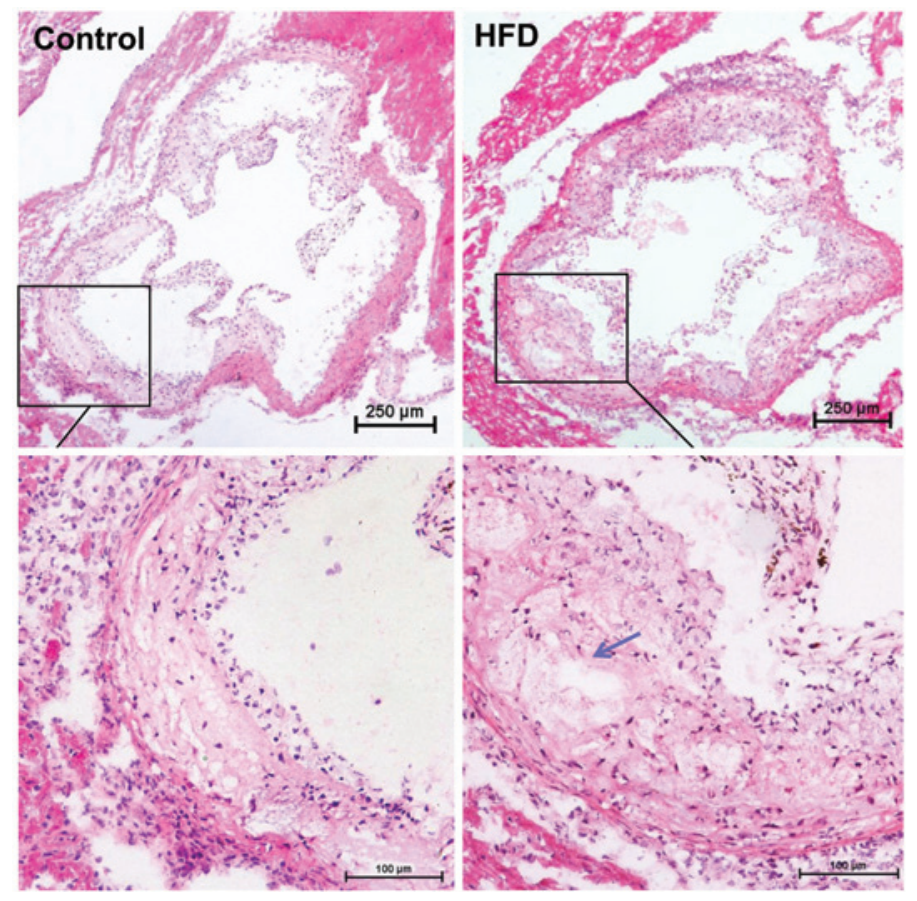

Figure 5. Histological analysis of hematoxylin and eosin-stained tissues. Foam cell-rich lesions were observed in the two groups of mice. A lipid core (arrow) was observed in the intimal lesions of the aortic root of $\mathrm{ApoE}^{-/}$mice in the HFD group. HFD, high-fat diet.

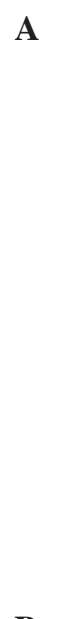

B
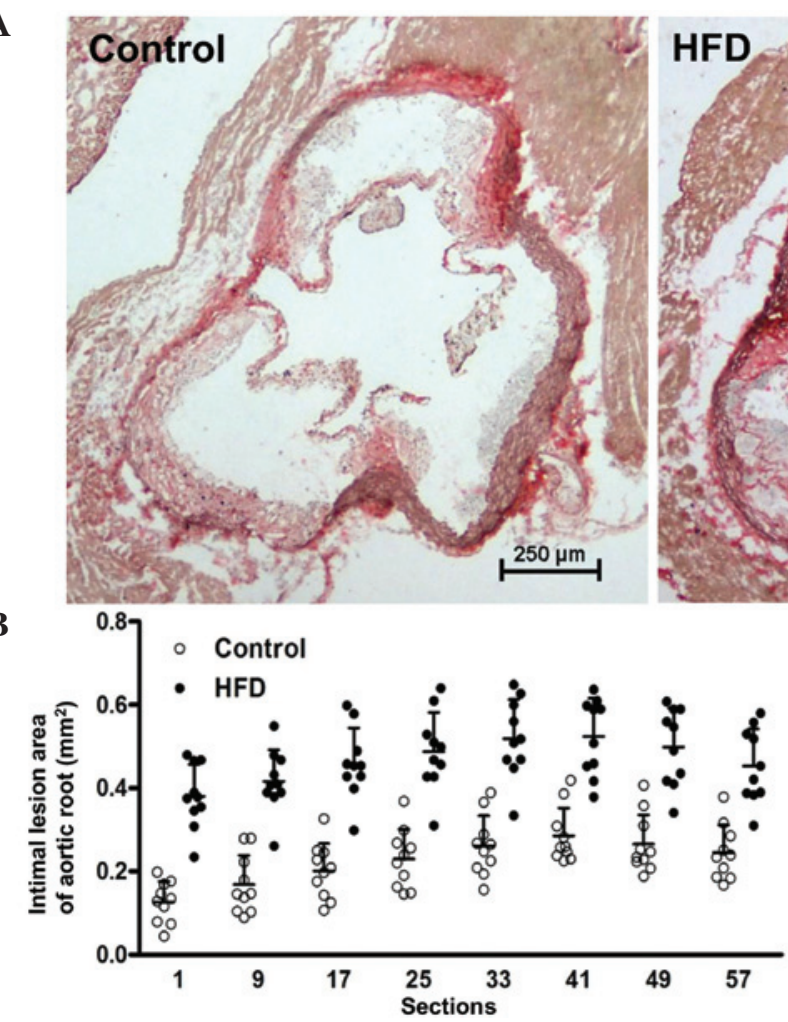

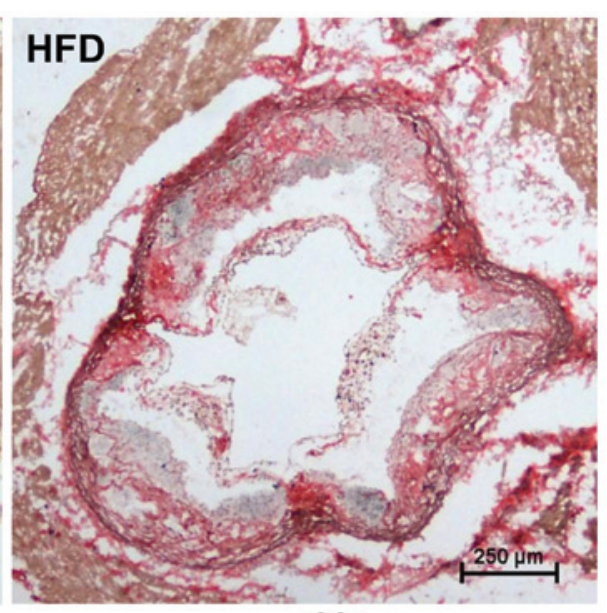

C

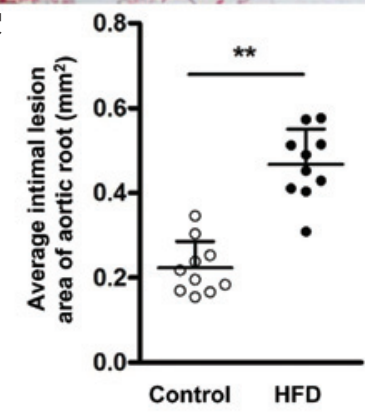

Figure 6. Microscopic analysis of the intimal lesions in the aortic roots. (A) Elastic van Gieson-stained cross sections of the aortic roots revealing the area of the lesions. (B) Sizes of the atherosclerotic lesion areas of eight individual cross sections of the aortic root of each mouse. (C) Average size of the atherosclerotic plaques in the aortic root. Data are expressed as the mean \pm standard deviation ( $\mathrm{n}=10$ for each group). ${ }^{* *} \mathrm{P}<0.01$, vs. control. HFD, high-fat diet.

with a HFD increased the total area of gross atherosclerotic lesions in apoE $\mathrm{E}^{-/-}$mice.

Analysis of microscopic lesions in the aortic root. Atherosclerotic lesions, predominantly composed of foam cells and macrophage-like cells, were observed in the aortic root of the two groups of apoE ${ }^{-/}$mice (Fig. 5). Following 12 weeks of feeding, no lesions containing only foam cells were observed in the $\mathrm{HFD}$ apoE $\mathrm{E}^{-/}$mice, but they remained present in the mice on the standard chow diet. Lipid cores were observed in the 

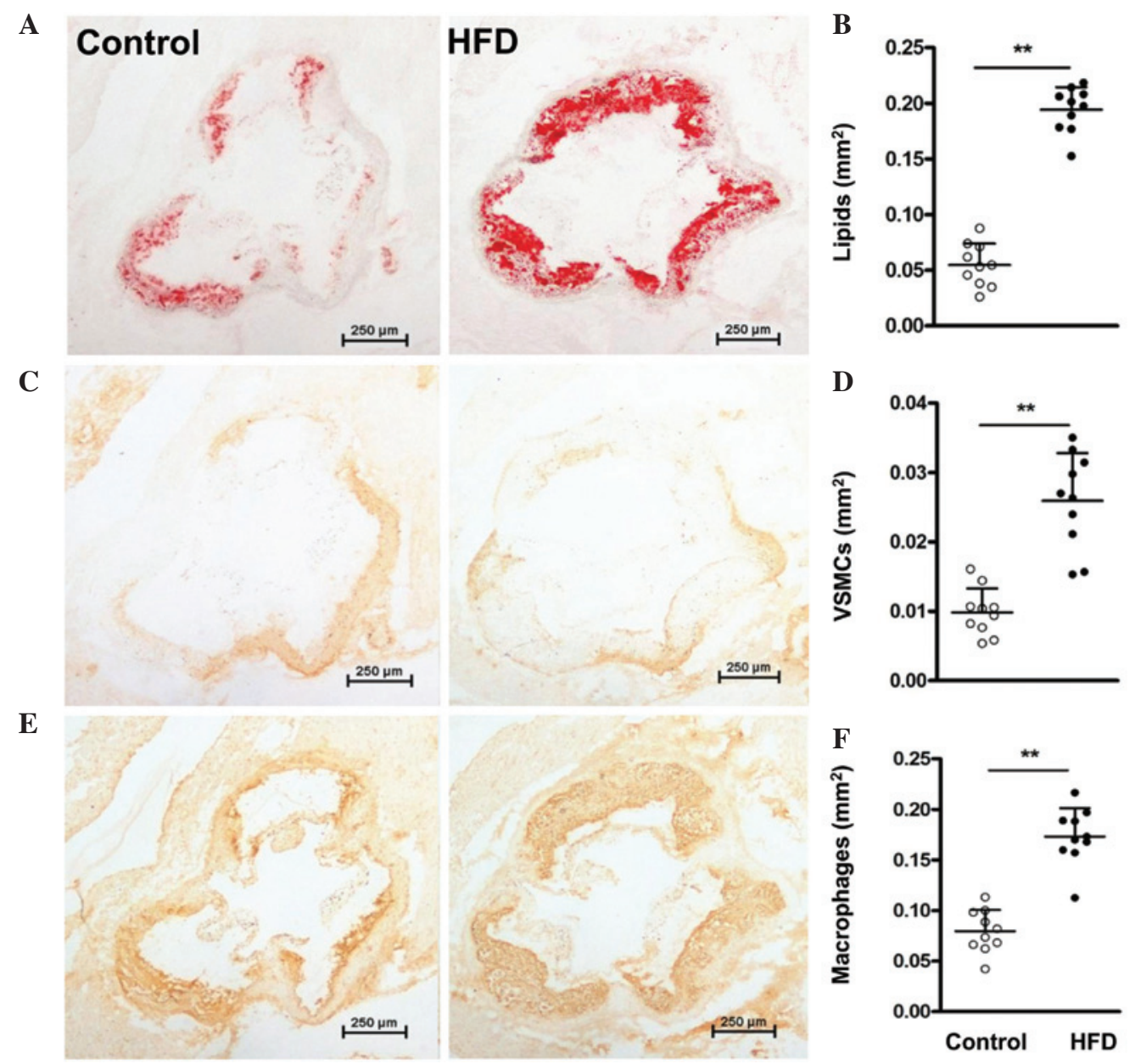

Figure 7. Microscopic analysis of the distribution of neutral lipids and other components of the lesions. (A) Oil Red O-stained cross sections of the aortic roots revealing the neutral lipids. (B) Size of the area occupied by neutral lipids. (C) Cross sections of the aortic roots, immunohistochemically labelled for VSMCs. (D) VSMC-positive area. (E) Cross sections of the aortic roots, immunohistochemically labelled for macrophages. (F) Macrophage-positive area. Data are expressed as the mean \pm standard deviation ( $\mathrm{n}=10$ for each group). ${ }^{* *} \mathrm{P}<0.01$, vs. control. VSMCs, vascular smooth muscle cells; HFD, high-fat diet.

intimal lesions of the aortic root of the HFD apoE ${ }^{-/}$mice (arrow in Fig. 5). The microscopic lesion area in the aortic roots was assessed using EVG staining (Fig. 6A). The HFD apoE ${ }^{-/-}$mice exhibited a significant increase in lesion area, compared with the control chow diet-fed apo $\mathrm{E}^{-/}$mice $(\mathrm{P}<0.01$; Fig. 6B and $\mathrm{C})$.

The foam cells and the lipid core in the atherosclerotic lesions were stained red using Oil Red O. The foam cells were widely distributed in the lesions (Fig. 7A). The area of the neutral lipids in the lesions was significantly wider in the HFD mice than in the control mice $(\mathrm{P}<0.01$; Fig. 7B). Immunohistochemical staining of $\alpha$-actin was used to identify vascular smooth muscle cells (VSMCs) in the atherosclerotic plaque of the aortic root (Fig. 7C). The two groups of mice expressed $\alpha$-actin on the surface of the plaques and in the aortic media. Macrophage expression in the atherosclerotic plaques was identified by immunohistochemical staining of galectin 3 (Fig. 7E). Macrophages were widely distributed in the lesions of the aortic roots in the two apoE $\mathrm{E}^{-/-}$mouse groups. HFD apoE $\mathrm{E}^{-1-}$ mice exhibited a significantly higher number of macrophages and VSMCs in their microscopic lesions, compared with the mice fed the standard chow diet $(\mathrm{P}<0.01$; Fig. 7D and F). In general, the mice, which were provided with the HFD developed more advanced lesions, compared with the mice, which were fed with the standard chow diet.

\section{Discussion}

Atherosclerotic lesions can develop either spontaneously or from HFD-induced hypercholesterolemia in genetically modified mice (7). These atherosclerotic lesions share several characteristic features of the atherosclerosis, which is observed in other animal models and humans $(8,22)$. Following the development of apoE ${ }^{-/-}$mice in 1992, these lesion-susceptible mice have been widely used in investigations of cardiovascular disease and pharmacodynamics $(5,23)$. Currently, there are no uniform international and domestic standards to evaluate the severity of atherosclerotic lesions in this mouse model.

A method for the quantification of atherosclerotic lesions in the mouse aorta was introduced by Tangirala et al (14), which used Sudan IV staining to measure the area of gross atherosclerotic plaques in the entire aorta, and H\&E staining to measure the intimal microscopic lesions in the aortic roots. Other studies have evaluated lesions through analysis of the aortic root alone $(9,10,12)$ and there remains no uniform standard for selecting the cross sections. Although this method is labor-intensive and time-consuming, it has been useful for evaluating the severity of atherosclerotic lesions in mouse models (7).

The present study provided a detailed description of the isolation of the mouse heart and arterial tree for systematic 
analysis of the gross area of the lesions in the entire aortic tree, microscopic measurement of the size of the intimal atherosclerotic lesions on the aortic roots and immunohistochemical quantification of key cellular components in these lesions. The method used to assess microscopic lesions in cross sections of the aortic roots recommended in the present study can be used to analyze the area of intimal lesions and can also analyze the composition of these atherosclerotic lesions. In addition, the present study compared the atherosclerotic lesions of apoE $\mathrm{E}^{-1-}$ mice fed a standard diet with those fed a HFD, by assessing the gross lesions in the entire aortic tree and microscopic lesions in the aortic root. The area of the lesions and the distribution of key cellular components, including VSMCs and macrophages, were simultaneously analyzed within these lesions. The results demonstrated that HFD gradually increased plasma levels of TC and TG in the apoE $\mathrm{E}^{-/-}$mice, and that the levels were significantly higher in the mice fed a HFD, compared with those fed a standard chow diet. The areas of the gross lesions in the entire aorta were larger in the HFD group than in the control group. As described in the results of the present study, the ratios of the area of gross atherosclerotic lesions to the luminal surface area of the entire aorta in all $\mathrm{apoE}^{-/-}$mice were low, however, extensive and severe atherosclerotic lesions were identified in the aortic roots. Analysis of microscopic lesions in the aortic root with serial cross sections of the heart, the results demonstrated that the HFD apoE $\mathrm{E}^{-/}$mice had more severe atherosclerotic lesions in the aortic root, compared with the apoE $\mathrm{E}^{-/-}$mice on a chow diet. In the present study, EVG staining was also used to quantify the area of the atherosclerotic lesions in the aortic root. EVG stains elastic fibers and, therefore, highlights the junction between the tunica intima and the tunica media (18). Compared with Oil Red O, which stains for neutral lipids, EVG enables easier and more accurate quantification of the lesion area (7). Oil Red $\mathrm{O}$ does not stain the entire lesion area in a section, due to the fact that the lesions contain nonlipid-engorged smooth muscle cells, extracellular matrix and non-neutral lipids, including unesterified cholesterol (7). Consequently, Oil Red O staining routinely underestimates the size of the lesions. Therefore, quantifying the lesion area frequently requires manual manipulation with image software to outline the lesions defined by the internal elastic lamina and the luminal boundary, which can be indicated by EVG staining (7).

Due to the fact that lesions in the aortic roots appear earlier than lesions elsewhere in the aorta in several genetically susceptible and genetically modified mice (7), cross-sectioning the heart makes it possible to assess lesion formation and the extent of the lesions following a relatively short dietary experiment, whereas analyzing the entire aortic tree subsequent to a relatively long dietary experiment may be more suitable for regression investigations, which require extensive atherosclerosis and more advanced lesions (14). One of the advantages of the former is that the cross sections can provide a basis for the three-dimensional (3D) reconstruction of lesions (14). Otherwise, following quantification of the area of gross atherosclerotic lesions using Sudan IV or Oil Red O staining, the entire aortic tree cannot be used in the analysis of the lesion components. Serially frozen cross sections of the heart, however, can be used to assess the atherosclerotic lesion area and the components of the lesions (24).
The drawbacks of this quantitative method are the difficulties in developing a standard and the technical challenges of the methods. The key step in this method is the determination of the first cross section, to ensure that analysis began at the same anatomical position for every mouse. In the present study, the cross section where the three aortic valve leaflets initially appeared together was selected as the initial point of analysis, and it was ensured that the distance between the appearance of the first aortic valve leaflet and the appearance of all three aortic valve leaflets together was $\leq 80 \mu \mathrm{m}$. A distance $\geq 80 \mu \mathrm{m}$, indicated an error in the slicing angle, which affects the reproducibility of the results. Two technical points that require mention are as follows: i) Mouse heart specimens were embedded in OCT, with the aortic arch positioned upwards, and their location was fixed during the process of freezing; ii) subsequent to the appearance of the first aortic valve, the angle of the specimen holder of the slicing machine was carefully adjusted and the slicing process was performed with caution. Using this method, eight cross-sections were collected at $64 \mu \mathrm{m}$ intervals on one glass slide, and a single similar glass slide for each mouse heart was sufficient for different types of histological analysis. This method markedly reduces workload, compared with the previously described method (12). Due to the fact that only PBS was perfused into the mice, other specific experimental procedures, including immunofluorescent staining that requires a special fixing method, can be used on the cross sections. However, the evaluation of atherosclerosis remains inadequate using this method alone. As lesions exist not only in the aortic root, but are distributed sporadically throughout the aorta and its principal branches, neither of the two methods provide a complete quantitative evaluation of atherosclerosis. Thorough quantitative assessment of murine atherosclerosis can be achieved by combining the two techniques. The extent of atherosclerosis in the aortic root or any other segment of the entire aorta alone does not reflect the severity of atherosclerosis in the entire aorta.

When possible, the quantitative method of analyzing both the gross atherosclerotic lesion area in the entire aortic tree and the microscopic lesions in the aortic roots, is accurate and thorough. This method can be applied to the investigation of the development of atherosclerotic lesions, and to examine the pathogenesis of atherosclerosis and molecular mechanism of cardiovascular drugs in mouse models. Current murine investigations can also use high-resolution micro-ultrasound imaging, high-resolution 3D magnetic resonance imaging and Fourier Transform Infrared spectroscopic imaging to quantitatively assess atherosclerotic lesions (25-27). These methods are noninvasive and may improve current understanding of atherosclerosis in humans.

\section{Acknowledgements}

This study was partly supported by the National Natural Science Foundation of China (grant. no. 81270348).

\section{References}

1. Daugherty A: Mouse models of atherosclerosis. Am J Med Sci 323: 3-10, 2002.

2. Kapourchali FR, Surendiran G, Chen L, Uitz E, Bahadori B and Moghadasian MH: Animal models of atherosclerosis. World J Clin Cases 2: 126-132, 2014. 
3. Zaragoza C, Gomez-Guerrero C, Martin-Ventura JL, Blanco-Colio L, Lavin B, Mallavia B, Tarin C, Mas S, Ortiz A and Egido J: Animal models of cardiovascular diseases. J Biomed Biotechnol 2011: 497841, 2011.

4. Knowles JW and Maeda N: Genetic modifiers of atherosclerosis in mice. Arterioscler Thromb Vasc Biol 20: 2336-2345, 2000

5. Plump AS, Smith JD, Hayek T, Aalto-Setälä K, Walsh A, Verstuyft JG, Rubin EM and Breslow JL: Severe hypercholesterolemia and atherosclerosis in apolipoprotein E-deficient mice created by homologous recombination in ES cells. Cell 71: 343-353, 1992.

6. Zadelaar S, Kleemann R, Verschuren L, de Vries-Van der Weij J, van der Hoorn J, Princen HM and Kooistra T: Mouse models for atherosclerosis and pharmaceutical modifiers. Arterioscler Thromb Vasc Biol 27: 1706-1721, 2007.

7. Daugherty A, Lu H, Howatt DA and Rateri DL: Modes of defining atherosclerosis in mouse models: Relative merits and evolving standards. Methods Mol Biol 573: 1-15, 2009.

8. Nakashima Y, Plump AS, Raines EW, Breslow JL and Ross R: ApoE-deficient mice develop lesions of all phases of atherosclerosis throughout the arterial tree. Arterioscler Thromb 14: 133-140, 1994

9. Mani S.Li H, Untereiner A, Wu L, Yang G, Austin RC, Dickhout JG, Lhoták S, Meng QH and Wang R: Decreased endogenous production of hydrogen sulfide accelerates atherosclerosis. Circulation 127: 2523-2534, 2013

10. Wang Y, Zhao X, Jin H, Wei H, Li W, Bu D, Tang X, Ren Y, Tang C and Du J: Role of hydrogen sulfide in the development of atherosclerotic lesions in apolipoprotein E knockout mice. Arterioscler Thromb Vasc Biol 29: 173-179, 2009.

11. Paigen B, Morrow A, Holmes PA, Mitchell D and Williams RA: Quantitative assessment of atherosclerotic lesions in mice. Atherosclerosis 68: 231-240, 1987.

12. Purcell-Huynh DA, Farese RV Jr, Johnson DF, Flynn LM, Pierotti V, Newland DL, Linton MF, Sanan DA and Young SG: Transgenic mice expressing high levels of human apolipoprotein B develop severe atherosclerotic lesions in response to a high-fat diet J Clin Invest 95: 2246-2257, 1995.

13. Véniant MM, Pierotti V, Newland D, Cham CM, Sanan DA Walzem RL and Young SG: Susceptibility to atherosclerosis in mice expressing exclusively apolipoprotein B48 or apolipoprotein B100. J Clin Invest 100: 180-188, 1997.

14. Tangirala RK, Rubin EM and Palinski W: Quantitation of atheroscleros in murine models: Correlation between lesions in the aortic origin and in the entire aorta and differences in the extent of lesions between sexes in LDL receptor-deficient and apolipoprotein E-deficient mice. J Lipid Res 36: 2320-2328, 1995.
15. Guide for the Care and Use of Laboratory Animals. US National Institutes of Health, Bethesda, 2011.

16. Chen Y, Zhao S, Huang B, Wang Y, Li Y, Waqar AB, Liu R, Bai L, Fan J and Liu E: Probucol and cilostazol exert a combinatorial anti-atherogenic effect in cholesterol-fed rabbits Thromb Res 132: 565-571, 2013.

17. Ford A, Al-Magableh M, Gaspari TA, Hart JL: Chronic NaHS treatment is vasoprotective in high-fat-fed ApoE(-/-) mice. Int J Vasc Med 2013: 915983, 2013.

18. Zhang C, Zheng H, Yu Q, Yang P, Li Y, Cheng F, Fan J and Liu E: A practical method for quantifying atherosclerotic lesions in rabbits. J Comp Pathol 142: 122-128, 2010.

19. Yu Q, Li Y, Wang Y, Zhao S, Yang P, Chen Y, Fan J and Liu E: C-reactive protein levels are associated with the progression of atherosclerotic lesions in rabbits. Histol Histopathol 27: 529-535, 2012.

20. de Boer RA, Voors AA, Muntendam P, van Gilst WH and van Veldhuisen DJ: Galectin-3: A novel mediator of heart failure development and progression. Eur J Heart Fail 11: 811-817, 2009.

21. Liu E, Kitajima S, Higaki Y, Morimoto M, Sun H, Watanabe T, Yamada N and Fan J: High lipoprotein lipase activity increases insulin sensitivity in transgenic rabbits. Metabolism 54: 132-138, 2005.

22. Meir KS and Leitersdorf E: Atherosclerosis in the apolipoprotein-E-deficient mouse: A decade of progress. Arterioscler Thromb Vasc Biol 24: 1006-1014, 2004.

23. Reddick RL, Zhang SH and Maeda N: Atherosclerosis in mice lacking apo E. Evaluation of lesional development and progression. Arterioscler Thromb 14: 141-147, 1994.

24. Tupin E, Nicoletti A, Elhage R, Rudling M, Ljunggren HG, Hansson GK, Berne GP: CD1d-dependent activation of NKT cells aggravates atherosclerosis. J Exp Med 199:417-422, 2004.

25. Ni M, Zhang M, Ding SF, Chen WQ and Zhang $Y$ : Micro-ultrasound imaging assessment of carotid plaque characteristics inapolipoprotein-E knockout mice. Atherosclerosis 197: 64-71, 2008.

26. McAteer MA, Schneider JE, Clarke K, Neubauer S, Channon KM and Choudhury RP: Quantification and 3D reconstruction of atherosclerotic plaque components in apolipoprotein E knockout mice using ex vivo high-resolution MRI. Arterioscler Thromb Vasc Biol 24: 2384-2390, 2004.

27. Wrobel TP, Mateuszuk L, Kostogrys RB, Chlopicki S and Baranska M: Quantification of plaque area and characterization of plaque biochemical composition with atherosclerosis progression in ApoE/LDLR $\left(/^{-}\right)$mice by FT-IR imaging. Analyst 138: 6645-6652, 2013. 